\title{
THIRD POSTER PAPER DISCUSSION
}

HANES: Welcome to the discussion of poster papers which fall under the somewhat fanciful title "Distant Globular Clusters". You will have seen in your inspection of these that in fact they represent quite a mixed lot. We have in total just over twenty papers to discuss and time is short, so let us begin. Our first paper is that of Zinnecker et al. There is a demonstrable consistency of colors between the nuclei and the extra-nuclear parts of some of the nucleated dwarfs in the Virgo cluster. On this basis, the authors suggest that Colin Norman's proposed mechanism of nucleus formation by mergers of clusters through dynamical friction may be credible. I have some small reservations about the results owing to the very difficult treatment for wavelength-dependent seeing in establishing nuclear colors. Comments and questions?

VAN DEN BERGH: At a given magnitude leve1, round dE galaxies have a higher probability of exhibiting nuclei than do flatter ones. This suggests that the nuclei might have been formed from low angular momentum gas.

ZINNECKER: I certainly agree that it is the rounder virgo dwarf ellipticals that tend to have nuclei. However, the reason for this tendency could be either that the small ellipticity (indicative of little rotation) gives nuclei a chance to form (as suggested by Sidney van den Bergh) or that the existence of a nucleus causes the dwarf ellipticals to become secularly more spherical (as advocated by Colin Norman on the basis of the scattering of radial orbits off the central density cusp). It appears to be a chicken and egg situation. I have discussed this in a recent paper presented at the Rome Astrophysical Colloquium 1986 .

DJORGOVSKI: If this merger picture is correct, then why do we see such nuclei in the diffuse dwarfs? They are the least luminous or least dense of all galaxies.

ZINNECKER: It seems that the low central mass density of the low surface brightness dwarf ellipticals allows star clusters to spiral to the center without being disrupted, while disruption will occur for galaxies with less shallow gravitational potentials.

SCHOMMER: George Lake and I looked at this dynamical timescale for the clusters in Fornax following a suggestion by Scott Tremaine some 10 years ago. We were worried that they all should be found in the nucleus, but when you treat the background density carefully, the dynamical friction time is $\sim 1 / 2$ Hubble time, which is consistent with the observed distribution.

HANES: Thank you. We move on now to a poster paper by Judy Cohen on 
the globular clusters of M 87. Her results have been discussed to a degree already particularly in John Huchra's review; let me just remind you that she finds small radii gradients in color both for the light and for clusters. More interestingly, she confirms strom et al.'s finding that the median cluster color at given radius is everywhere bluer than the galaxy light, although she, in fact, started as a skeptic on this very point!

COHEN: The published version of this work will have luminosity functions and spatial distributions as well for three galaxies, NGCs 4406,4472 and 4486 .

LEE, H. M.: The color difference between globular clusters and underlying light may be consistent with the scenario of making the halos by destroying the globular clusters. The stars leaving the clusters are lower mass stars than those staying in the clusters.

TRIMBLE: What is the ratio of total Milky Way halo turnoff to that in the globular clusters?

VAN DEN BERGH: $100-1$.

TRIMBLE: If so, then you cannot account for the field halo population, as a small factor of material shed from clusters. You need 998 of the total population, and so this cannot account for the field cluster color difference.

HANES: We now turn our attention to a paper by Lauer and Kormendy, one already published. They find the globular cluster distribution in $M 87$ to have a core an order of magnitude larger than that exhibited by the underlying light, and argue that this difference is innate. Comments?

LAUER: The core is large. We assume that the core is primordial only because we don't know how to make it now. We also believe that dynamic friction cannot make the observed nucleus by the central accretion of clusters; it fails by at least a factor of 10 .

VAN DEN BERGH: Grillmair Pritchet and van den Bergh, find no radii variation of the luminosity function of globular clusters within the inner few arcmin. of $M 87$.

HANES: This would seem to confirm that there are no strongly mass-dependent disruptive processes as a function of radius within M 87 .

HANES: Our next contribution by Held et al., reports $U$ photometry of the globular clusters in M 87. These authors have detected the core alluded to in the previous paper, and perhaps I should recall here, that Rene Racine recognized the value of the $U$ bandpass in sharpening the contrast of the clusters against the galaxy: a decade ago he detected the central surface density plateau on a 200-inch U plate. 
Held et al. have nicely confirmed and quantified the effect, and will in due course present us with a fully calibrated $U$ luminosity function.

We turn now to an exciting contribution by Thompson and Valdes: the discovery of globular clusters around the giant elliptical NGC 4874 in the Coma cluster. In his review talk this morning, Bill Harris also recorded success in this difficult endeavor, but there are some differences: Bill sees a central concentration, which Thompson and Valdes do not, and quotes a specific frequency of $S-30$, while Thompson and Valdes find $S \sim 2$. Are these discrepancies worrying?

THOMPSON: The specific frequency determined by Frank Valdes and me for NGC 4874 is a local value and not a global value. In situations where the cluster population is more extended than the galaxy light, the local specific frequency increases as a function of radius. The global value mentioned by Bill Harris this morning is the most significant value scientifically but both values are "correct".

HARRIS, W.: I'd like to add first that Laird Thompson and I were aware of each other's results before the conference and the points of agreement were enough to make us each more willing to present them! My CCD material was taken at the prime focus of the CFHT with NGC 4874 centered on the chip, and so covers a wider field than Laird's material (but with lower resolution). I used the detected population in a rather wid: annulus around 4874 and scaled it to an annulus of the same linear size around $M 87$, assuming Coma to be 5.5 times farther away than Virgo.

COHEN: Are you convinced that you have overcome the effects of clustering in the field of background galaxies?

HARRIS, W.: We're well aware of the problems involved in subtracting off background to find a residual excess of objects, and it's true that the density of faint background galaxies can easily vary by factors of 508 or so from one place to another. However, the detection of the cluster system in Coma is a factor of 3 or 4 above the background level at $B>25$ and is not at all a marginal detection.

HUCHRA: Would either of you guys be brave enough to quote a distance modulus?

HARRIS, W.: No. The fit of the Coma luminosity function to that of $M 87$ is just a consistency fit and not a distance measurement.

HANES: Thank you. We next examine the presentation by Harris, Bothun and Hesser of the results of their search for globular clusters associated with edge-on spiral galaxies. Some systems have been detected, the most interesting being in NGC 7814, where the galaxy halo and the globular cluster system are both flattened perhaps by rotation. I invite questions. 
SCHOMMER: I have a question concerning $S$ vs. luminosity and environment. While Bill Harris this morning didn't speak about the late-type disk or irregular galaxies, we have in the Local Group a wide range of galaxy luminosities with well studied cluster systems. And the number of clusters increases steadily, so $S$ remains reasonably constant.

HARRIS, H.: It is difficult to estimate the specific frequency $S$ for spirals. When the projected cluster distribution differs from the spheroid light, a radius must be chosen at which to calculate $S$, or else assumptions must be made about the cluster distribution in the uncounted (central and outer) regions in order to estimate the total cluster population. In the late-type spirals NGC 5170 and NGC 5907, no central bulge is visible, and in all edge-on spirals decomposition of an exponential disk, thick disk, bulge and/or halo is difficult and model-dependent.

SCHOMMER: Do we know of any other group yet, which has been searched over a wide range of galaxy luminosities, or is virgo the test case, at least for a cluster environment?

HARRIS, W.: I can't think of any other groups like the Local Group which have a similar or wider range of galaxy types and sizes, to measure cluster populations. It might be best to go right to Virgo to get a complete sample.

HANES: Hugh Harris has made the important point that the specific frequency $S$ is at best ill-defined: do we normalize the cluster population against total galaxy mass, halo mass, or (perhaps equivalently) total or halo luminosity? For these disk systems in particular meaningful intercomparisons of $S$ are difficult.

Globular clusters have also been detected in the so galaxy NGC 3115 and a luminosity function has been reported in a poster paper by Held and Capaccioli. It is exciting to see these results appear for so many new cluster samples. Would the authors care to comment?

HELD: We have not yet carried out tests for magnitude-dependent imcompleteness, and our luminosity function must be regarded as preliminary.

HANES: You do appear to reach the turnover in that function, which may provide a useful test of the universality of the globular cluster luminosity function.

That remark leads me naturally to our last "extragalactic" poster paper, by myself and Whittaker. We argue on the basis of Monte Carlo simulations that maximum likelihood statistical methods will permit globular cluster luminosity functions to be used as distance indicators to a precision of about 108 out to at least distance moduli of 32 mag from the ground. It is not even necessary to reach the turnover, 
provided the intrinsic shape of the luminosity function is indeed universal. Of course we would like as much reassurance as possible on that point!

STATLER Any claim that you can get $\mathrm{H}_{\mathrm{o}}$ to 108 deserves to be challenged: your method depends on the assumption that the width of the distribution, $\sigma$, be universal. At the bottom of your poster you say that NGC 3379 suggests this may be true. Can you elaborate on this?

HANES: NGC 3379, a classical EO galaxy, is the only one which has been studied deeply enough to permit the acid test. For it, $\sigma=0.9 \pm 0.3$ formally, according to Pritchet and VandenBergh. This value is consistent with the value $(\sigma \simeq 1.2)$ seen both in the Milky Way and in M 31. (The large error bar is because the sample is numerically small.)

LAUER: You say $\sigma=0.9 \pm 0.3$ for $\mathrm{N} 3379$, but for $\mathrm{M} 87 \sigma=1.8$. These are both ellipticals but they do not have universal $\sigma$.

HANES: I do not in fact claim $\sigma=1.8$ for $M$ 87; rather, I point out that within the uncertainties a range of $\sigma$ values fit the deep vandenBergh et al. counts. My concern is that crowding, non-randomness in the background field, and the necessary incompleteness corrections at $B>24$ ay deform the observed function. I go into details in the submitted paper.

GRINDLAY: Would not a better use of your maximum liklihood technique be to establish the universality of the Gaussian form of the luminosity function and its value of sigma rather than to use it as a distance indicator?

HANES: That would be ideal but deriving $\sigma$ really requires going well past the peak - it's not well constrained. It is very important to do this in a number of galaxies! For other galaxies, where we see the bright tail only, the only thing one can sensibly do is adopt $\sigma$ and derive a distance.

KING: This morning the use of a Gaussian luminosity function made me uncomfortable; now you are really scaring me, because you rely so much more on the detailed accuracy of a Gaussian. Let's remember that Hubble's original luminosity function for galaxies was also Gaussian.

HANES: That's true, but the census of globular clusters in the Milky Way is quite different from the galaxy data Hubble worked with a half century ago. Moreover, we have deep complete functions in the $M 31$ halo. But the Gaussian merely represents an empirical fit, which works very well; I do not claim any special reason for its use other than practicality. It may eventually need modification. 
We11, we must move on - back into our own Galaxy in fact, for a series of papers on color-magnitude diagrams. The first of these is by Samus, who presents photographic photometry for several globular clusters. One concern $I$ have is that of calibration: two clusters are reported to have a 4 magnitude gap between the horizontal branch and the turnoff, and reference is made to a third, $w$ Cen, discussed by Cannon. But Cannon withdrew that conclusion, which was based on some simple error; Don VandenBergh has shown that a value of 3.5 mag seems to be nearly universal. Would the author care to comment?

SAMUS: I am not as strong as I used to be in my opinion that ages do differ, though I am not alone in regarding age as a possible second parameter. Photographic photometry can still be of some use, especially if we measure many more stars than do the observers using CCD's.

HANES: Certainly the panoramic detectors do provide very large samples, and we welcome these results.

Our next paper, by Buonanno et al., presents photometry for 10,000 stars in $M 3$. Interestingly, one sees here that the $A G B, H B$, and RGB stars all follow the mean luminosity distribution while the blue stragglers are less (rather than more) concentrated. This argues against their being mass-exchange binaries, which might settle towards the cluster center. I invite comments.

FUSI PECCI: Using plates it was impossible to get highly accurate photometry down to the turnoff in the central region of the cluster. This implies that we have no direct information on the presence, number, and spatial distribution of the blue stragglers there. Within the anulus we measured, BSs seem to be more frequent in the outer regions, contrary to the evidence found in NGC 5466 (a very loose cluster studied even in the very central region). A detailed comparison and discussion will be however possible only after the complete coverage of the central regions of $M 3$ we are currently obtaining with CCD cameras.

KING: I hope that you will use this material to make an explicit study of crowding corrections.

FUSI PECCI: Crowding has been of course a problem in the reductions and we made many tests and checks to reduce and evaluate its influence. We don't believe that crowding might have affected at any significant level the projected spatial distribution of blue stragglers we have derived.

HANES: In an attractive presentation Richer and Fahlman have described the study of three fields in $M 5$ at 8,21 , and 58 core radii. The similarity in $C M$ diagrams argues against any metallicity gradient, and the luminosity functions are likewise similar to $V=23$. Differences fainter than that may bespeak mass segregation. 
RICHER: The evidence for mass segregation at the faint end must be still regarded as somewhat tentative because (1) the number of stars entering into the luminosity function for the outer field is quite small and (2) the incompleteness correction for the inner field is large (but well determined).

HANES: No further comments? Then I invite discussion of Piotto et al.'s study of 3,000 stars in $M 30$. Those authors find consistency with McClure et al.'s suggestions: this low metallicity cluster seems to have a steep luminosity function, although their data do not go quite deep enough to provide absolute confirmation.

ORTOLANI: Our points are limited in magnitude but reliable due to the large number of stars.

RICHER: Fahlman and I have a luminosity function for $M 30$ done with a CCD at CFHT and the slope of the fitted power low mass function is very flat, in fact the best fit is $X=0.0$. This should not necessarily be taken as a counter example to the McClure et al. result that the more metal poor the cluster the steeper the mass function ( $M 30$ is very metal poor.)

VANDENBERG: Regarding the consistency of observed LF's with the McClure et al. (1986) result that the slope of the mass function varies inversely with $[\mathrm{Fe} / \mathrm{H}]$, it is important to note the following. Theoretical LFs show a turnup at the faint end $M_{y} \sim 7.5$, depending on metallicity) due to a flattening of the mass-luminosity relation independent of the shape of the mass function. Therefore, if a turnup is observed, then it may be reasonable to assume that the present day mass function is similar to the IMF. Certainly, if a turnup is not observed, then there must have been significant evaporation of 1ow mass stars. Therefore, to test whether the observed cluster LFs follow the McClure et al. relation, they must be extended to sufficiently faint magnitudes. Since the $M 30$ data do not reach $M_{v}=7$, it is premature to conclude that it is an exception to the rule.

MCCLURE: Is it not true Harvey that the incompleteness corrections in your last points are very large?

RICHER: Its a factor of 3 in the last point...but that can be dropped without changing the result at all.

MCCLURE: A factor of 3 correction is very large!

VAN DEN BERGH: If memory serves we correctly the $M 30$ has the bluest integrated (B-V) color of any luminous globular cluster. A reddening as large as $E_{B-V}=0.13$ would make its intrinsic (B-V)。 color quite unusual. 
HANES: I now draw your attention to an automated photometric study by Trimble and Irwin of NGC 6809. I can claim to have had a hand in this, since I took the AAT plates used in their work, which was accomplished using the APM in the other Cambridge. The derived main sequence is wide enough to admit the existence of binaries of the $w$ UMa type.

TRIMBLE: We set out to look for main sequence variables and found about eight candidates, several of which were found independently by $M$. Hazen. They have periods consistent with $w$ UMas. These should be followed up for light curves, but this is not likely to be done by any of us involved in the project.

HANES: I propose to discuss the next two papers together: each of them (one by Ortolani, the other by Noble et al.) reports a study of $\omega$ Cen. Ortolani presents tantalizing hints of the detection of white dwarfs in $w$ Cen, although I am puzzled that these occur in only one of his fields. The other study, based in part on 1-meter observations from South Africa, reports that the main sequence of $w$ Cen is wide below the turnoff; in this way it is like the giant branch, the width of which is well established.

ORTOLANI: Only one field (the innermost one) was fully reduced. The number of possible white dwarfs is consistent with theoretical predictions. Quasar contamination could be a problem.

NOBLE: I'd like to reiterate the intrinsic width of the main sequence, in this study shown to be present to below $V=20$. Subsequent data to much fainter limits $(V-24)$ confirm this spread to those limits. These deeper data do not show white dwarfs, although they do reach the region where the very brightest would show up.

HANES: Thank you. Alcaino and Liller suggest, in our next paper, that age determinations for globular clusters are best made through use of $\mathrm{V},(\mathrm{B}-\mathrm{I})$ color magnitude diagrams. Isochrones are now available which incorporate the redder bandpasses, and their figures show that the shallower form of the turnoff makes fitting the data formally easier.

Da COSTA: I point out that CCD's have wide spectral response which often is not taken advantage of as much as it should be.

PENNY: I tried some (B-V) and (B-I) work and found little difference in resultant accuracy. I agree that $(V-R)$ is a good regime.

Da costA: I find that observations in $B$ and $R$ give better precision than observing $B, V$. The errors in $V$ and $R$ are usually comparable, but for given change in $T_{\text {eff }}$, the change in $(B-R)$ is almost twice as large as for $(B-V)$.

HESSER: Don VandenBerg has emphasized the importance of matching isochrones in the subgiant region, yet in the 47 Tuc CM diagram shown 
the Alcaino/Liller data appear to cross the isochrones. Do you see that effect in any of your data?

Da COSTA: I cannot really comment, as $I$ do not have any data of precision to equal that of Hesser and Harris' V, (B-V) data for 47 Tuc.

COHEN: You don't win using broad band photometry using CCD's beyond $r$. The atmospheric emission in the near infrared accessible to CCD's is so great that you cannot go deeper or get more accurate photometry than if you stay below $7500 \AA$.

HANES: Our last observational paper, by Buonanno et al., reports a study of cluster ages. These authors have dereddened and deblanketed cluster CMDs. Subsequent main sequence fitting of the clusters, one with another, then reveals the dependence of HB luminosity on metallicity, which in turn (via $\Delta \mathrm{V}_{\mathrm{TO}}^{\mathrm{HB}}$ ) yields cluster ages. They find ages of $~ 16 \mathrm{Gyr}$, with no significant spread.

BUONANNO: I want to remind you that, as there is no evidence of varying $\Delta \mathrm{V}_{\mathrm{TO}} \mathrm{HB}$, constant ages for globular clusters imply the existence of the Sandage effect.

HANES: Finally, we turn our attention to several papers of a more theoretical nature. Bhatia has carried out $\mathrm{N}$-body simulations of clusters in eccentric orbits around a point mass galaxy to aid our understanding of cluster disruption. Any comments?

BHATIA The expansion of a cluster depends on the eccentricity of the orbit. For $e=0.6$ the cluster disrupts while for $e=0.65$ the cluster expands. Perhaps this expansion can explain sparse clusters in the halo.

HANES: Next, I draw your attention to a contribution from Takayanagi, who has assumed that globular clusters trace the mass of a galaxy and that the distribution is best matched by polytropes of index 5 to derive masses for various galaxies. However, I am puzzled that he remarks upon the inadequacy of de Vaucouleurs-type $\left(r^{1 / 4}\right)$ fits to the cluster distributions: my experience is that such fits are very successful.

Finally, then, I invite comments on a program described in a poster by Ramamani. N-body simulations of clusters within an isothermal galaxy will be studied. I gather that this is a report on work in progress?

RAMAMANI: Due to problems with energy conservation in the modified code, the results could not be presented. I hope to publish these in the near future.

HANES: Then we wish you success and look forward to seeing those results in due course. 
And my thanks to all the participants - authors and attendees for their evident interest in the poster papers we have been discussing this afternoon. We have seen some exciting results. 